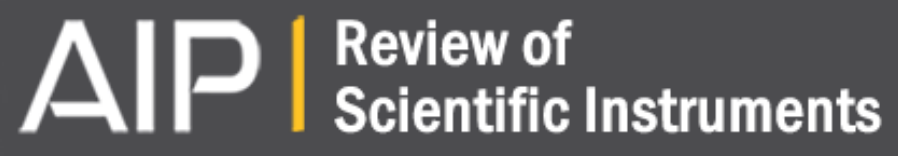

\section{Mobile setup for synchrotron based in situ characterization during thermal and plasma-enhanced atomic layer deposition}

Jolien Dendooven, Eduardo Solano, Matthias M. Minjauw, Kevin Van de Kerckhove, Alessandro Coati,

Emiliano Fonda, Giuseppe Portale, Yves Garreau, and Christophe Detavernier

Citation: Review of Scientific Instruments 87, 113905 (2016); doi: 10.1063/1.4967711

View online: http://dx.doi.org/10.1063/1.4967711

View Table of Contents: http://scitation.aip.org/content/aip/journal/rsi/87/11 ?ver=pdfcov

Published by the AIP Publishing

\section{Articles you may be interested in}

Ag films grown by remote plasma enhanced atomic layer deposition on different substrates

J. Vac. Sci. Technol. A 34, 01A126 (2016); 10.1116/1.4936221

A modular reactor design for in situ synchrotron x-ray investigation of atomic layer deposition processes Rev. Sci. Instrum. 86, 113901 (2015); 10.1063/1.4934807

In situ synchrotron based $x$-ray techniques as monitoring tools for atomic layer deposition

J. Vac. Sci. Technol. A 32, 010801 (2014); 10.1116/1.4851716

In situ synchrotron based x-ray fluorescence and scattering measurements during atomic layer deposition: Initial growth of $\mathrm{HfO} 2$ on $\mathrm{Si}$ and Ge substrates

Appl. Phys. Lett. 98, 231905 (2011); 10.1063/1.3598433

In situ synchrotron $\mathrm{x}$-ray characterization of $\mathrm{ZnO}$ atomic layer deposition

Appl. Phys. Lett. 97, 191904 (2010); 10.1063/1.3514254

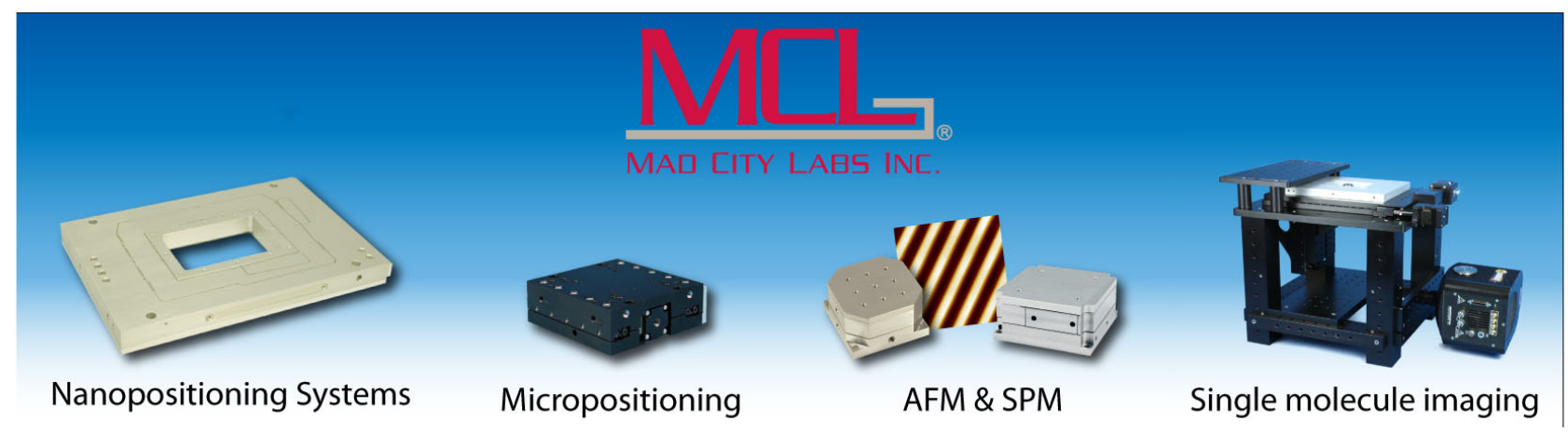




\title{
Mobile setup for synchrotron based in situ characterization during thermal and plasma-enhanced atomic layer deposition
}

\author{
Jolien Dendooven, ${ }^{1, a)}$ Eduardo Solano, ${ }^{1}$ Matthias M. Minjauw, ${ }^{1}$ Kevin Van de Kerckhove, ${ }^{1}$ \\ Alessandro Coati, ${ }^{2}$ Emiliano Fonda, ${ }^{2}$ Giuseppe Portale, $\left., 3, b\right)$ Yves Garreau, ${ }^{2,4}$ \\ and Christophe Detavernier ${ }^{1}$ \\ ${ }^{1}$ Department of Solid State Sciences, COCOON, Ghent University, Krijgslaan 281/S1, B-9000 Ghent, Belgium \\ ${ }^{2}$ Synchrotron SOLEIL, L'Orme des Merisiers, Saint-Aubin, BP48, 91192 Gif-sur-Yvette, France \\ ${ }^{3}$ DUBBLE Beamline BM26, ESRF European Synchrotron, Avenue des Martyrs, CS40220, \\ 38043 Grenoble, France \\ ${ }^{4}$ Matériaux et Phénomènes Quantiques (MPQ), Université Paris Diderot-Paris 7, Sorbonne-Paris-Cité, CNRS, \\ UMR 7162, 10, rue A. Domon et L. Duquet, 75205 Paris Cedex 13, France
}

(Received 12 September 2016; accepted 31 October 2016; published online 16 November 2016)

\begin{abstract}
We report the design of a mobile setup for synchrotron based in situ studies during atomic layer processing. The system was designed to facilitate in situ grazing incidence small angle $\mathrm{x}$-ray scattering (GISAXS), x-ray fluorescence (XRF), and x-ray absorption spectroscopy measurements at synchrotron facilities. The setup consists of a compact high vacuum pump-type reactor for atomic layer deposition (ALD). The presence of a remote radio frequency plasma source enables in situ experiments during both thermal as well as plasma-enhanced ALD. The system has been successfully installed at different beam line end stations at the European Synchrotron Radiation Facility and SOLEIL synchrotrons. Examples are discussed of in situ GISAXS and XRF measurements during thermal and plasma-enhanced ALD growth of ruthenium from $\mathrm{RuO}_{4}$ (ToRuSTM, Air Liquide) and $\mathrm{H}_{2}$ or $\mathrm{H}_{2}$ plasma, providing insights in the nucleation behavior of these processes. Published by AIP Publishing. [http://dx.doi.org/10.1063/1.4967711]
\end{abstract}

\section{INTRODUCTION}

Atomic layer deposition (ALD) is a vapor-based thin film deposition technique. ${ }^{1,2}$ The self-limited surface reactions enable atomic level thickness control and exceptional conformality when depositing nanocoatings on complex 3D-shaped surfaces. ${ }^{3}$ Over the past decade, these unique advantages have rendered ALD a mainstream deposition technique in microelectronics. In recent years, the reverse process of atomic layer etching (ALE) through self-limited surface reactions is gaining increasing attention. ${ }^{4}$ During both ALD and ALE, plasma exposure has been shown beneficial, as the presence of plasma species enables more versatile and reactive surface chemistries for atomic layer processing at low substrate temperatures. ${ }^{4,5}$

Over the past decade, in situ characterization during ALD has been instrumental towards achieving a fundamental understanding of the physico-chemical phenomena that are controlling ALD. ${ }^{6}$ In essence, atomic layer processing is controlled by surface chemistry, and therefore any technique for operando physico-chemical characterization of the nearsurface region can be highly relevant. Although traditional surface science techniques such as X-ray photoelectron spectroscopy (XPS), low-energy ion scattering (LEIS), or scanning probe microscopy (SPM) seem at first sight most appropriate,

\footnotetext{
a) Email: Jolien.Dendooven@ugent.be. Tel.: +329264 8572. Fax: +329264 4996.

b) Present address: Zernike Institute for Advanced Materials, University of Groningen, Nijenborgh 4, 9747 AG Groningen, The Netherlands.
}

they suffer from a "pressure gap," in the sense that ALD requires intermittent exposures to precursor vapors and gases in the $10^{-3}-10$ mbar range, making it difficult to integrate ALD into setups for surface spectroscopy or microscopy that often require ultra-high vacuum operation. The conformal nature of ALD tends to result in significant coating of the chamber walls and any detector or other expensive and sensitive parts that are exposed within the vacuum chamber. Therefore, although recently several groups reported exciting data from near ambient pressure XPS, ${ }^{7,8}$ traditional UHV-based surface science techniques have been mainly implemented "in vacuo" instead of "in situ" for ALD research so far.

For in situ studies, quartz crystal microbalance (QCM), mass spectrometry, and optical characterization techniques such as infrared spectroscopy and spectroscopic ellipsometry (SE) have been most popular., ${ }^{6,9}$ In particular the optical techniques have proven to produce reliable and reproducible data. They can be relatively easily implemented on any ALD reactor where an optical path is available towards the substrate, by introducing windows that are transparent to the relevant part of the electromagnetic spectrum. When using $\mathrm{KBr}$ windows, illuminating the surface of the growing film with infrared light enables vibration spectroscopy to identify and monitor the presence of molecular fragments on the surface during film growth. When using quartz windows, polarized light in the visual or UV part of the electromagnetic spectrum can be reflected off the surface of the growing film. Analysis of the change in polarization through optical modelling provides information on key properties of the growing film, such as thickness, refractive index, roughness, resistivity, etc. In recent 
years, several groups have explored extending this concept of using light to study ALD growth towards the X-ray part of the electromagnetic spectrum. ${ }^{8,10-23}$ Indeed, when an ALD reactor is equipped with x-ray transparent windows (e.g., beryllium, graphite, or kapton), then the film can be (intermittently) exposed to $\mathrm{x}$-rays during growth, and a wide range of $\mathrm{x}$-ray based thin film characterization techniques can be used for in situ characterization, as recently reviewed by Devloo-Casier et $a l .{ }^{17}$ Since ALD is typically used for nanocoatings with a thickness of 0.1 to several tens of nanometers, while $\mathrm{x}$ rays typically penetrate several micrometers deep into most materials, it is challenging to obtain a sufficient signal to noise ratio for $\mathrm{x}$-ray based characterization techniques, in particular when targeting acquisition rates of 1-60 $\mathrm{s}$ in order not to interfere too much with the standard exposure cycle of the ALD process. Therefore, while lab-based $x$-ray sources are used routinely for $e x$ situ analysis of, e.g., the crystallinity of ALD grown films, the in situ studies typically require the high photon flux that can only be offered by synchrotron based sources. To enable such synchrotron based experiments, a dedicated ALD setup is required that is preferably mobile so that it can be used at different beamline end stations (enabling different x-ray based characterization techniques), preferably at different synchrotron user facilities. In recent years, "in situ" setups have been developed at the National Synchrotron Light Source (NSLS I) at Brookhaven National Lab (by temporarily transforming a multi-purpose UHV chamber at beamline X21 into an ALD reactor), ${ }^{10,24}$ at the Pohang Light Source (PLS), ${ }^{14}$ at the Advanced Photon Source (APS) at Argonne National Laboratory, ${ }^{21}$ at Stanford Synchrotron Radiation Lightsource (SSRL) ${ }^{20}$ and at SOLEIL, ${ }^{19,22}$ while "in vacuo" setups have been developed at BESSY $\mathrm{II}^{25,26}$ and at SSRL, ${ }^{27,28}$ mainly targeting XPS.

Here, we report the design of a dedicated mobile reactor for in situ characterization during ALD of nanocoatings at synchrotron facilities. The setup enables $x$-ray fluorescence (XRF), x-ray absorption spectroscopy (XAS), and grazing incidence small angle scattering (GISAXS) measurements during both thermal and plasma-enhanced ALD. In situ XRF has proven an easy-to-implement and valuable tool to monitor ALD growth at the synchrotron. ${ }^{10,11,13,14,29}$ It provides direct information about the amount of material deposited per ALD cycle, on planar substrates as well as on more complex 3D surfaces. In situ XAS during ALD allows probing the local atomic environment and provides information such as the level of oxidation, coordination numbers, and bond lengths. ${ }^{12,16,19}$ GISAXS is a scattering technique used to study the morphology of nanoscale objects at surfaces, interfaces, or in thin films. ${ }^{30}$ In ALD research, in situ GISAXS has been applied to investigate conformal deposition in porous thin films ${ }^{18}$ and on a layer of semiconducting quantum dots. ${ }^{23}$ In addition, GISAXS is greatly suitable for monitoring the morphological evolution during ALD of noble metals. ${ }^{20,31,32}$ In these processes, including ALD of ruthenium, the growth is usually initiated in localized islands spread across the surface, which give rise to a scattering pattern in GISAXS that can be analyzed to extract information about the island dimensions and arrangement on the surface. Only in a later growth stage, the islands coalesce into a continuous layer which, depending on the density of nuclei, may exhibit a rough surface morphology. This presents a problem for the application of ALD to deposit, for example, ruthenium as capacitor electrode in DRAM devices or as a seed layer for $\mathrm{Cu}$ electroplating, motivating the need for studying the initial growth stages of noble metal ALD processes.

In this paper, we first give a detailed description of the setup and how it has been successfully installed at the DUBBLE BM26B, ${ }^{33}$ SixS, and SAMBA ${ }^{34}$ beamlines at the European Synchrotron Radiation Facility (ESRF) and SOLEIL synchrotrons. To illustrate the functionality, examples will be discussed of in situ XRF and GISAXS measurements during ALD growth of ruthenium.

\section{INSTRUMENT DESIGN}

\section{A. Design requirements}

A first key goal was to achieve a truly mobile setup, which can be used both "at home" in a lab environment as well as for "in situ" experiments at multiple beamline end stations at multiple synchrotron facilities. Since synchrotrons are user facilities, experiments are usually scheduled for several days up to maximum one week, including the time required for setting up all required equipment at the beamline end station. To enable efficient use of the allotted beamtime, it was considered a crucial design constraint that the ALD process of interest can be implemented and qualified in the ALD setup prior to the beamtime in a more traditional labbased environment, and that the ALD setup can then be installed and the process of interest re-qualified at the beamline end station in a limited setup time of maximum one day. A second key design goal concerned to achieve a setup that is compatible with existing reactor designs and cycle times used in research style ALD reactors, so that the acquired in situ data are relevant for standard processing conditions and can be easily cross-compared with the literature data and results from lab-based experiments. In view of the technological relevance of plasma-enhanced approaches during atomic layer processing, the addition of a plasma source was considered a key requirement, enabling in situ characterization during not only thermal but also plasma-enhanced ALD processes. A final key goal concerned flexibility. Therefore, it was decided to target a multi-technique chamber, enabling the use of different x-ray based characterization techniques. We targeted optimized geometries for GISAXS, XRF, and XAS, including both $\mathrm{x}$-ray absorption near edge structure (XANES) and extended x-ray absorption fine structure (EXAFS). In particular the implementation of the GISAXS technique had important repercussions on the requirement for invacuum shielding and slits, which will be discussed in detail.

\section{B. Overview of the mobile ALD reactor design}

Based on the above criteria, it was decided to implement a compact design based on a standard pump-type ALD reactor with a remote inductively coupled plasma source, as reported by Rossnagel et al. ${ }^{35}$ and commercially implemented by, e.g., 
Oxford Instruments and Cambridge Nanotech. The choice for a high vacuum setup with a turbo pump enables to achieve a base pressure of $10^{-7} \mathrm{mbar}$, which is beneficial for ensuring system integrity by easily detecting possible leaks in the chamber or gas lines, which is advantageous from a safety point of view. The possibility to perform ALD at low system pressures is also advantageous for plasma-enhanced ALD, which is typically performed at $10^{-3}-10^{-2}$ mbar. Moreover, the presence of a turbo pump enables complete evacuation of the chamber in between the precursor vapor or reactant gas exposure during the ALD process. For systems that rely on purging for removing the remaining vapor or gas, maintaining a small chamber volume and ensuring efficient gas flow by avoiding cavities and other spaces where gas could get trapped is a key requirement to avoid excessive purge times, and these put severe restrictions on the overall size and shape of the chamber. For a pump-type system, these requirements are relaxed, which enables more degrees of freedom during chamber design, facilitating the implementation of, e.g., the necessary windows for enabling in situ characterization.

The ALD chamber is essentially a six-way CF100 cross, with eight additional flanges-two CF63 and six CF40 flanges-pointing towards the optical center of the cross (Figure 1(a)). The bottom flange was intentionally kept flat and acts as a support plate for mounting the chamber onto sample positioning devices at the synchrotron. The overall

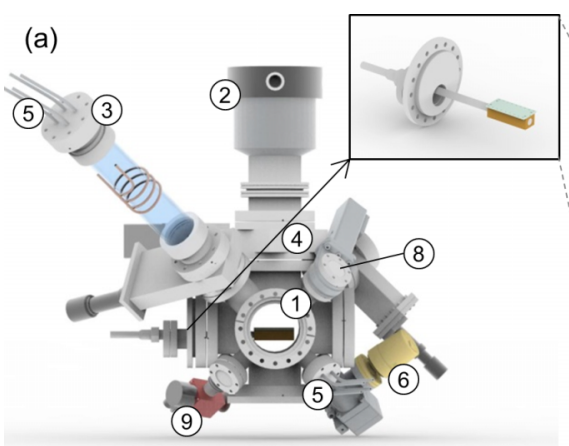

(c)

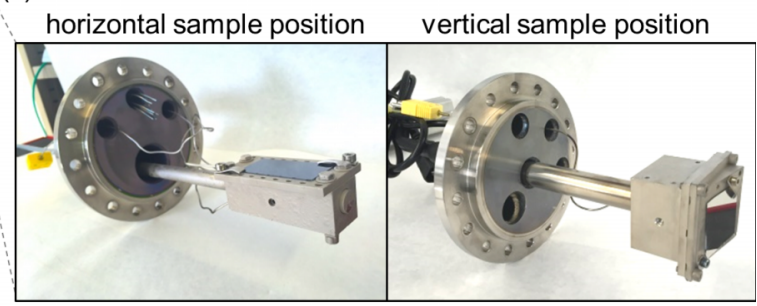

(d)
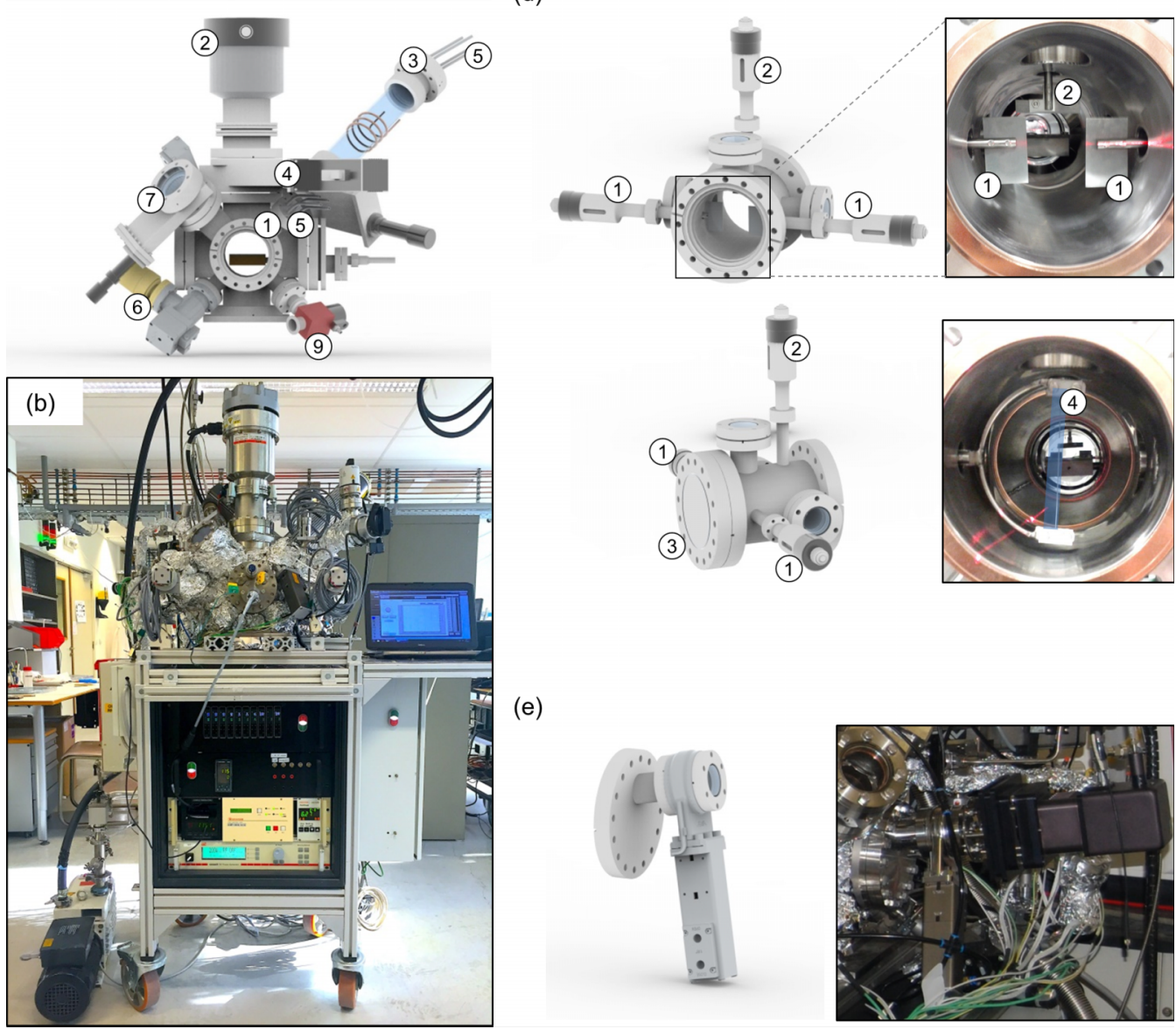

(e)

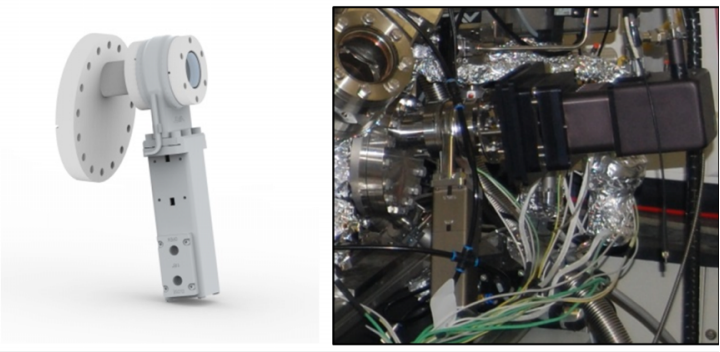

FIG. 1. (a) Overview of the mobile ALD reactor with (1) ALD chamber, (2) turbo pump, (3) RF plasma source, (4) gate valve, (5) precursor and gas inlets, (6) valve-protected pressure gauge, (7) valve-protected optical view port, (8) valve-protected CF35 Be window, (9) bypass valve to the roughing line. (b) Picture of the ALD reactor installed on a mobile 19 in. electronics rack. (c) Drawing and pictures of the dedicated flanges with horizontal or vertical sample stage and incorporated sample heating. (d) Drawing and pictures of the CF100 extension tube with motorized anti-scattering slits (1) and knife edge (2), and mounted beryllium window (3). At the exit site a beam stop (4-highlighted in blue) is installed in vacuum. (e) Drawing and picture of the side flanges used for in situ SE measurements. 
dimensions of the cross were selected to be compatible with both Huber tilt stages (2-circle segment 5203.20) as well as typical dimensions of hexapods that are often used at beamline end stations for sample alignment and that are here generally referred to as "diffractometer." In essence, the most restricting requirement here was that the sample-to-baseplate distance should not exceed the rotational center-to-baseplate distance of $170 \mathrm{~mm}$ for the Huber system (2-circle segment 5203.20).

Figure 1(a) shows some of the essential components that enable (plasma-enhanced) ALD such as the turbo pump, the plasma source, the precursor and gas inlets, and the pressure gauge. Depending on the geometrical constraints of the experiment, one of the vertical side flanges can be occupied with a dedicated flange that acts as substrate holder with integrated substrate heating (Figure 1(c)), as discussed in detail in Section II F. The other side flanges are used for mounting either $\mathrm{x}$-ray transparent windows with optional anti-scattering systems for experiments at the synchrotron (Figure 1(d) and Section II E) or quartz windows for in situ SE measurements in the lab (Figure 1(e)). These interchangeable side flanges allow for a lot of flexibility when designing experiments.

To ensure mobility, the setup was kept compact so that it could be mounted on top of a standard mobile 19 in. electronics rack (Figure 1(b)). In addition to its function as a mobile mounting platform for supporting the ALD reactor in the lab or during transport to the synchrotron, the mobile rack also contains all relevant control electronics and can stay in the radiation hutch in close proximity to the ALD reactor.

\section{System installation at the synchrotron}

Using overhead cranes that are typically available in most beamline end stations, the chamber can be lifted off the mobile rack and onto a diffractometer. The bottom flange of the chamber consists of a flat stainless steel plate with fixed mounting holes. When installing the chamber at a beamline end station, a custom adaptor plate is designed with one set of mounting holes that are compatible with the local diffractometer and a second set of holes that are compatible with the bottom flange of the ALD reactor, thus ensuring solid mounting of the ALD reactor onto the diffractometer. By mounting the entire chamber (including turbo pump, precursor bottles, and plasma source) onto a diffractometer, we avoid the need for motorized sample movement within the reactor, which would complicate the design. All precursor bottles are positioned close to the main chamber and directly connected to it through fixed stainless steel tubes. The compact design, where basically a complete ALD reactor is installed onto a diffractometer, enables precursors to be installed and tested in the lab prior to a synchrotron campaign. Since the entire reactor can then be lifted in one piece onto the diffractometer, there is no need to reassemble the chamber or to reestablish vacuum/gas connections when installing the setup at the synchrotron, enabling installation in about $24 \mathrm{~h}$, while the diffractometer enables careful sample positioning and alignment with respect to the synchrotron beam.

\section{Vacuum system}

The chamber can be pumped down using a combination of a rotary vane pump (Pfeiffer DUO 20M) and a turbo pump (Edwards STP301). For stability reasons, the turbo pump is mounted on the top flange of the chamber, to reduce off-axial loading of the diffractometer. A gate valve (VAT Series 108) is mounted in between the chamber and the turbo pump, which can be closed to enable high pressure ( $>10^{-2} \mathrm{mbar}$ ) operation of the chamber (static exposure mode). Computer controlled valves arrange for either rough pumping of the chamber by the rotary pump through a bypass-line or direct pumping through the gate valve using the turbo pump. The pressure in the chamber can be monitored using a vacuum gauge (Pfeiffer PKR 251), which can be protected during deposition using a computer controlled angle valve. The pressure in the backing line of the turbo pump is continuously monitored using a Pirani pressure gauge (Pfeiffer TPR 280).

\section{E. X-ray windows and anti-scattering systems}

One CF35 and two CF100 beryllium windows with thicknesses of 0.2 and $1 \mathrm{~mm}$, respectively, were purchased from Materion Electrofusion (PF-60, unpolished). Alternatively, double kapton windows can be used, with differential pumping in between both windows to enable a sufficiently low base pressure in the main chamber. ${ }^{19}$ A CF100 beryllium window is mounted on one of the side flanges as an entrance window for the synchrotron beam. For XRF and XAS measurements, the $0.2 \mathrm{~mm}$ thick CF35 beryllium window is used as an exit window and is either installed on a vertical CF100 to CF35 reducer flange or on one of the $\mathrm{CF} 35$ corner flanges pointing to the center of the chamber (Figure 1(a), see also Section III). Coating of this window during exposure of the sample to the ALD reactants is prevented by means of a protecting mini gate valve (VAT Series 010). In GISAXS experiments, the scattered $\mathrm{x}$-rays from the sample leave the chamber through the second CF100 beryllium window (Figure 2(a)). In addition, dedicated CF100 extension tubes with integrated motorized slits were designed for GISAXS. When the x-ray beam hits the first beryllium window to enter the chamber, a lot of scattered and diffracted radiation is being generated because of interaction with the window. Therefore, inside the vacuum chamber just behind the beryllium window, we implemented a set of horizontal and vertical motorized slits made of tungsten to block this spurious radiation, so that only the main transmitted beam can interact with the sample surface (Figures 1(d) and 2(a)). After interacting with the sample, both the diffuse scattered signal (which is of interest) as well as the specularly reflected beam take off from the sample towards the exit beryllium window. In all GISAXS setups, the specular reflection and direct beam need to be blocked by a wire- or needle-like shield to prevent damage to the detector. Although this is often done by a motorized shield in front of the detector (outside the vacuum chamber), it is important to avoid interaction between the intense direct and specularly reflected beam and the exit beryllium window, since this generates scattered radiation that generates an unwanted background that gets overlaid on top of the pattern of scattered 
(a)
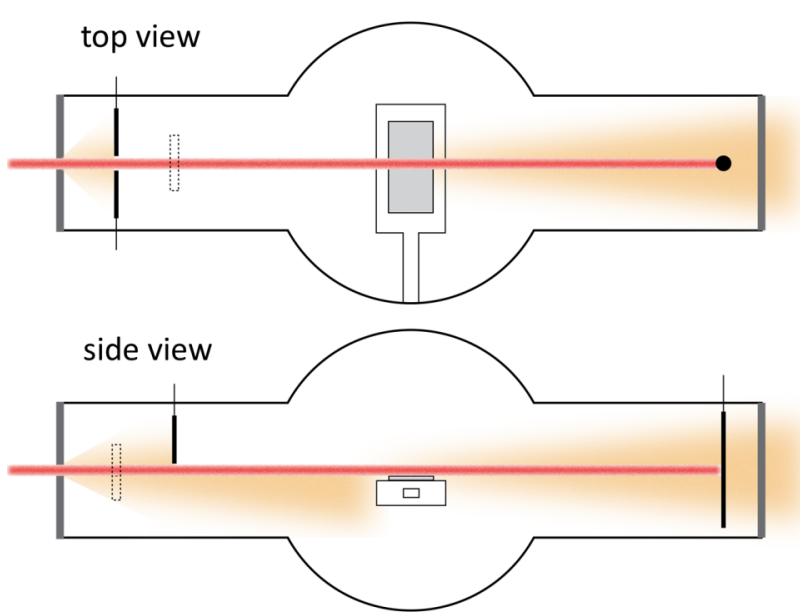

(b)
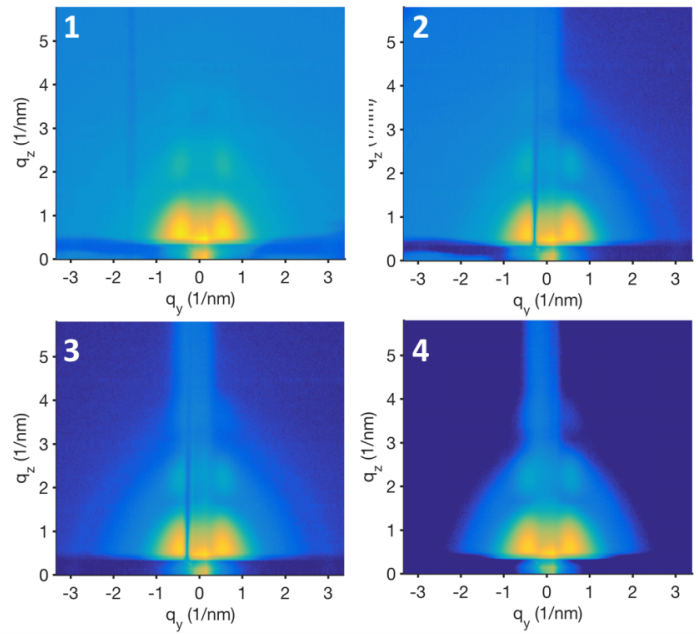

FIG. 2. (a) Schematic representation of the beam path (from left to right) in a GISAXS experiment with incident beam in red, scattered x-rays in orange, and anti-scattering slits, knife edge, and beam stop in black. (b) GISAXS pattern measured on a Pt/Si sample (1) without slits and beam stop, (2) with left slit, (3) with left and right slits, and (4) with left and right slits and beam stop. The scattering originates from the particulated Pt morphology.

radiation that originates from the sample. Therefore, in a CF100 extension tube that can be added in between the sample and the exit window, a motorized tungsten wire $(1 \mathrm{~mm}$ diameter) was mounted to avoid the specular reflection to reach the beryllium window by getting completely absorbed in the wire (Figures 1(d) and 2(a)).

The importance of in vacuum anti-scatter slits is illustrated by the GISAXS data shown in Figure 2(b). The sample consisted of ALD-grown Pt nanoparticles on $\mathrm{Si}$ (deposited using 60 cycles of the $\mathrm{MeCpPtMe}_{3} / \mathrm{O}_{3}$ ALD process ${ }^{36}$ ). The first image shows a GISAXS pattern that was acquired without any anti-scatter feature in place. Although the scattering features from the sample are clearly visible, the data suffer from considerable background. Subsequent images show the effect of positioning the right and left front slits (i.e., the vertical slits in between the entrance window and the sample) to define the incoming beam and of positioning the wire to block the specular reflected beam. The final image clearly has a much improved background, revealing much more detailed features in the scattering pattern from the sample that would otherwise have been completely lost in the high background signal.

\section{F. Sample stage and sample heating}

The design choice to incorporate all aspects related to mounting the sample, sample heating, and measurement of the sample temperature onto a single flange allows for a lot of flexibility when designing experiments, since different substrate mounts and different approaches to sample heating can be easily incorporated by designing different sample flanges to enable experiments over different temperature ranges or in different experimental geometries. Two examples of substrate holder flanges will be discussed here. A first example concerns a flange that enables horizontal mounting of samples for GISAXS and XRF experiments during ALD (Figure 1(c), left). The sample can be mounted onto a molybdenum plate using small clamps. The sample plate is supported by a copper block clamped onto a stainless steel tube that is welded onto the sample flange. The stainless steel tube can be fitted with a cartridge heater and a thermocouple. All electrical connections for the heaters and the thermocouple are performed in air, thus avoiding any electrical shorting during, e.g., ALD of metallic coatings. Sample temperatures of up to $375^{\circ} \mathrm{C}$ can be easily and reliably achieved using a Eurotherm temperature controller (Model 2408). A second substrate holder flange enables vertical mounting of samples for XAS measurements at the SAMBA beamline (Figure 1(c), right). The need for vertical mounting during XAS will be discussed further on. The method for heating and measuring the temperature of the sample is identical to the previous case. By designing an appropriate flange with dedicated heater, the setup can be easily extended to, for example, in situ experiments during high temperature sample annealing in a controlled ambient at pressures between $10^{-7}$ and $10^{3}$ mbar.

\section{G. ALD precursor and gas/plasma supply system}

Precursors and reactant gases are connected to the chamber using stainless steel tubes and standard VCR and Swagelok type connections. Figure 3 shows a schematic representation of the gas handling during ALD experiments.

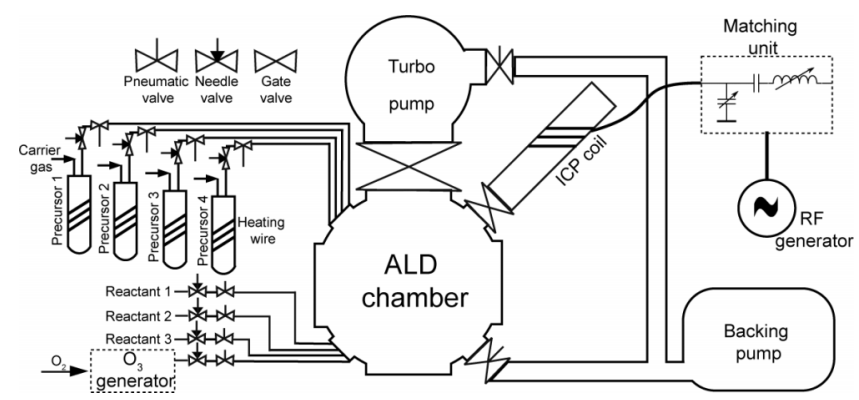

FIG. 3. Schematic representation of the ALD reactor and precursor and gas supply system. 
Pulses of precursor vapors and reactant gases are admitted into the chamber through a combination of manual needle valves and computer controlled Swagelok valves. As indicated, the setup has provisions for 4 precursor bottles. Ozone can be used by placing a mobile ozone generator (OzoneLab OL100) between the oxygen gas bottle and one of the inlets for the reactant gas. To avoid over-pressurizing the x-ray transparent windows, it is important to only connect gases using high-precision pressure regulators that are reducing the pressure from the gas bottle to slightly above 1 atmosphere. Additionally, a Swagelok one-way check valve has been installed on the chamber as an additional way of preventing over-pressurization.

To prevent precursor condensation, heating of the chamber walls and the precursor delivery lines is a critical aspect of any ALD reactor design. We have adapted a low-cost and flexible approach by using heating wires (Horst) and using aluminum foil to enhance the temperature uniformity along the stainless steel tubing and chamber walls. A thermocouple was firmly attached to the different heated parts of the chamber, and Omron temperature controllers were used to regulate the temperature of the different heated parts by regulating the electrical power delivered to the different heating wires.

To enable plasma-enhanced ALD, an inductively coupled plasma source was incorporated onto one of the diagonally downward oriented CF63 side flanges of the chamber. The plasma source consisted of a quartz tube with a three-turn copper coil. A $13.56 \mathrm{MHz}$ radio frequency (RF) Advanced Energy power supply and matching network were used to energize the plasma.

Most of the gate valves, angle valves, and precursor valves for controlling the pumping and gas flow into the chamber are pneumatically controlled. Computer control was achieved through a CompactRIO system of National Instruments with 32 digital output channels that were programmed through LabVIEW.

\section{H. Control software}

A key aspect of the control software concerns the interaction between the ALD control software and the beamline computer, which controls the sample (i.e., chamber) alignment and data acquisition. Since the chamber is used at different beamlines and different synchrotron facilities, a flexible solution was required, which does not involve adaptation of the control software on the beamline computer. The "screen" command in Unix offered an elegant solution, as this command enables text input into a Unix command prompt from multiple terminal screens, and even for external systems to hook into a running terminal window through a secure $\mathrm{SSH}$ connection. By initiating the screen command on the beamline computer and establishing a SSH connection between the laptop that is controlling the ALD reactor and the beamline computer, simple ASCII strings can be sent through this SSH connection and appear in the terminal window of the beamline computer as though they would have been typed onto the keyboard of the beamline computer. In this way, the LabVIEW program that is running the ALD experiment can send ASCII text to the beamline computer, triggering the movement of specific motors or the acquisition of data at specific moments during the ALD process. In essence, this enables us to control the entire experiment from the ALD controller without the need for any special software or hardware triggering from the beamline computer. The fact that the entire experiment is controlled from a single laptop also ensures data integrity and correct timing of the diffractometer movements and data acquisition.

\section{Summary of key design choices}

In summary, the setup is in essence a compact pump-type plasma-enhanced ALD reactor. Key adaptations for enabling synchrotron-based experiments consist of the following:

(1) Keeping the system compact, with an on-axis weight distribution, to enable easy installation at beamline end stations by simply lifting the complete setup from its mobile rack onto the diffractometer.

(2) The design choices of a flat baseplate (enabling easy positioning of the chamber onto the baseplate of the diffractometer), positioning the turbo pump along the $\mathrm{z}$-axis above the sample (ensuring a symmetrical loading of the diffractometer), and an appropriate sample-tobaseplate distance have ensured compatibility and easy installation onto standard hexapods and Huber tilt stages that are used at many beamline end stations.

(3) To enable x-rays to penetrate into the chamber, beryllium windows were mounted. It was a design decision not to incorporate welded beryllium windows, but instead to use standard CF-based components as offered by Materion Electrofusion. This offered the advantage that the beryllium windows only need to be mounted during synchrotron campaigns, while the side flanges can be replaced by regular $\mathrm{CF}$ flanges with quartz windows for enabling in situ SE when the mobile reactor is operated under standard lab conditions.

(4) To enable high quality GISAXS data, the in vacuum motorized slits proved a key requirement.

(5) Dedicated software was developed to enable full control of the experiment from the laptop that is running the LabVIEW program controlling the ALD process. By sending ASCII strings through an $\mathrm{SSH}$ connection to the active terminal on the beamline computer, the ALD controller can initiate motor movement or trigger the detector to acquire data at the appropriate time in between ALD cycles.

\section{EXAMPLES OF INSTALLATION AT SYNCHROTRON BEAMLINES}

Figure 4 illustrates 4 different implementations of the mobile reactor setup.

A first implementation (Figure 4(a)) is optimized for lab-based operation, where the beryllium windows are not mounted, and replaced by stainless steel flanges with smaller CF35 quartz windows at an angle of $\sim 12^{\circ}$ with respect to the sample surface plane for enabling SE measurements during 


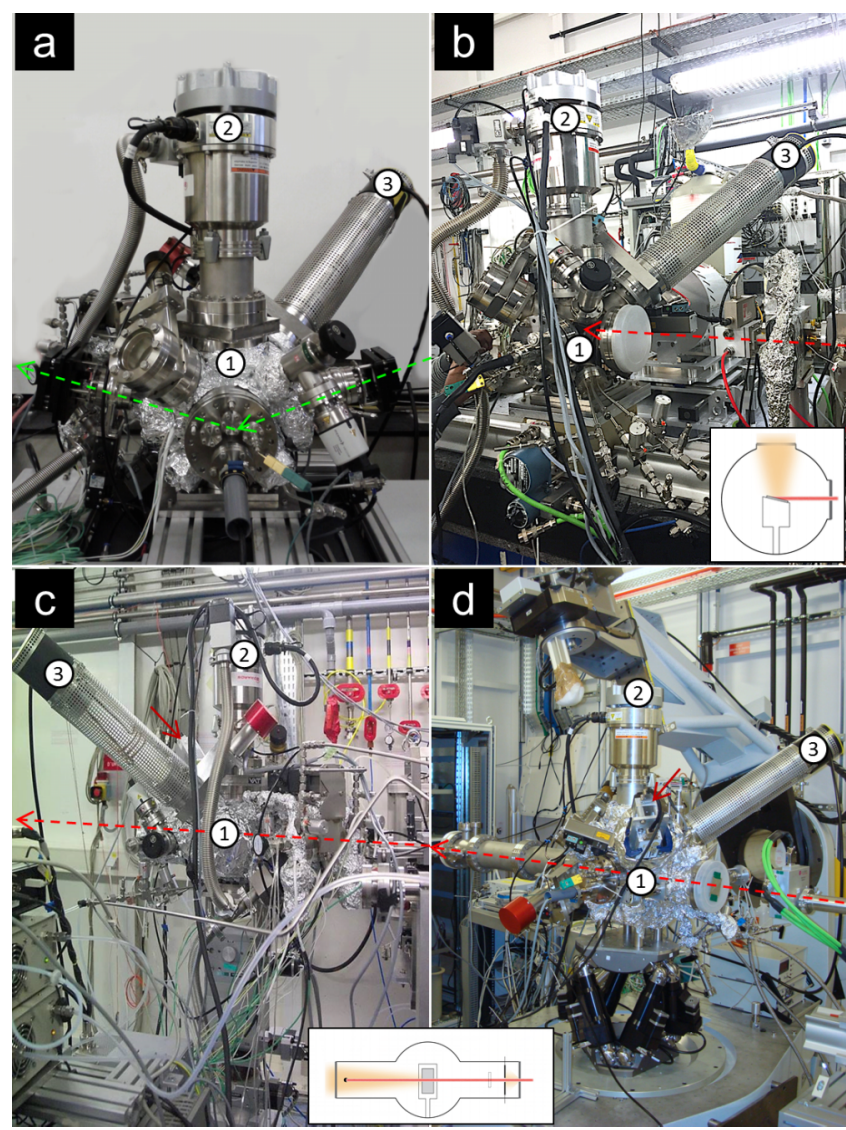

FIG. 4. Pictures of the mobile ALD setup installed (a) in the ALD lab at Ghent University (SE geometry), (b) on the x-y-z stage of the Samba beamline at SOLEIL (XAS geometry), (c) on the Huber pillar of the DUBBLE BM26B beamline at the ESRF (XRF/GISAXS geometry), and (d) on the hexapod of the SixS beamline at SOLEIL (XRF/GISAXS geometry) with (1) ALD chamber, (2) turbo pump, and (3) RF plasma source. The green and red dashed arrows indicate the path of the visual/UV light and X-ray beams, respectively. The red solid arrows in (c) and (d) indicate the position of the silicon drift detector used for XRF. The insets show a schematic top view of the sample position and beam path.

ALD (see also Figure 1(e)). The black parts in Figure 4(a) are the mounting platforms for the SE light source and detector, respectively.

A second implementation (Figure 4(b)) is optimized for in situ XAS measurements during ALD at the SAMBA beamline at SOLEIL. The use of a large Ge-based XRF detector (Canberra) requires that the samples are mounted vertically in the chamber. The incoming beam enters the chamber through a CF-100 beryllium window and illuminates the sample surface, causing fluorescence that is being emitted from the sample and transmitted through a CF-35 beryllium exit window and then detected by the Ge-based energy sensitive detector. The beam path is schematically indicated in the inset of Figure 4(b). An example of XAS data that was acquired using this geometry can be found in Ref. 19.

A third implementation (Figure 4(c)) is optimized for in situ GISAXS and XRF measurements at the DUBBLE BM26B beamline at ESRF. At this end station, the chamber can be mounted onto a pillar that consists of dedicated Huber parts enabling precise $x-y-z$ and tilt movements. The pillar ends in a flat baseplate, on top of which the mobile
ALD reactor can be mounted through a dedicated adaptor plate. The incoming x-rays enter the chamber through a CF100 beryllium window. Within the chamber, the motorized horizontal and vertical slits are installed to block scattered radiation from reaching the sample. The sample itself is mounted onto the horizontal sample stage with integrated sample heater. The reflected beam is blocked by the motorized tungsten wire inside the chamber, as discussed previously. The scattered x-rays can exit the chamber through a CF100 beryllium window. Immediately after the beryllium window, a configurable evacuated flight path can be installed that is part of the beamline, to avoid air scattering as the x-rays are on their way to the 2D Pilatus $1 \mathrm{M}$ detector (Dectris). Fluorescent radiation from the sample is being monitored through a CF35 beryllium window that is aimed towards the optical center of the chamber. For detecting the XRF signal, an energydispersive silicon drift detector (Vortex) is used.

A fourth implementation (Figure 4(d)) is optimized for in situ GISAXS and XRF measurements at the SixS beamline at SOLEIL. It is essentially identical to the previously discussed implementation at DUBBLE, but here a flight tube of up to $1 \mathrm{~m}$ is directly attached at the exit side of the vacuum chamber, in between the chamber and the CF100 extension with motorized in vacuum beam stop. For aligning the sample in the beam and for tilting the sample during GISAXS measurements, a hexapod is used. GISAXS measurements are recorded using a 2D CCD camera (MarCCD) or an X-ray pixel area detector (XPAD), while XRF measurements are performed with an energy-dispersive silicon drift detector (Vortex or Röntek).

\section{SELECTED RESULTS}

The in situ XRF and GISAXS capabilities of the mobile ALD setup are demonstrated with results obtained for thermal and plasma-enhanced ALD growth of ruthenium at the SixS beamline at SOLEIL. Ru was deposited using the inorganic $\mathrm{RuO}_{4}$-precursor in a reduction chemistry with $\mathrm{H}_{2}$ gas $^{37}$ or $\mathrm{H}_{2}$ plasma ${ }^{38}$ as reactants. The liquid $\mathrm{RuO}_{4}$ precursor dissolved in a methyl ethyl fluorinated solvent $\left(<0.5\right.$ wt. \% of $\left.\mathrm{RuO}_{4}\right)$ was contained in a stainless steel recipient manufactured and filled by Air Liquide S.A. The precursor container was put at a stabilized temperature of $30^{\circ} \mathrm{C}$ and connected to the chamber via a heated $\left(45^{\circ} \mathrm{C}\right)$ stainless steel tube. A thermal [plasmaenhanced] ALD cycle consisted of a $12 \mathrm{~s}$ [25 s] Ru precursor pulse, followed by a pumping period of $25 \mathrm{~s}$ to evacuate the chamber before the introduction of $\mathrm{H}_{2}\left[\mathrm{H}_{2}\right.$ plasma] for $10 \mathrm{~s}$ [12 s], which is again followed by a chamber evacuation (35 s [20 s]). During the Ru precursor pulse, the pressure in the chamber increased to $9 \times 10^{-3}$ mbar. For the $\mathrm{H}_{2}$ pulse, a static exposure mode was applied at a pressure of 6 mbar. The $\mathrm{H}_{2}$ plasma was ignited at a pressure of $1.3 \times 10^{-2}$ mbar using $250 \mathrm{~W}$ RF power. The substrate temperature was $100^{\circ} \mathrm{C}$ in all experiments. During the precursor and reactant pulses, a fast $\mathrm{x}$-ray shutter prevented the $\mathrm{x}$-rays from entering the chamber, avoiding possible interaction of the beam with chemical species. XRF and GISAXS measurements were performed with an acquisition time of $40 \mathrm{~s}$ after the last pumping step of an ALD cycle at an incidence angle of $1^{\circ}$. The beam energy 
was set to $5 \mathrm{keV}$, the lowest accessible beam energy at the SixS beamline, to allow for an efficient excitation of the Ru L lines at approximately $2.6 \mathrm{keV}$.

\section{A. In situ XRF}

In situ XRF was used to compare the growth rate on $\mathrm{H}$-terminated $\mathrm{Si}$ vs. $\mathrm{SiO}_{2}$. The $\mathrm{Si}-\mathrm{H}$ surface was obtained using an $\mathrm{HF}$ clean, while the oxidized $\mathrm{Si}$ surface was obtained by in situ $\mathrm{O}_{2}$ plasma cleaning of a $100 \mathrm{~nm}$ thermally grown $\mathrm{SiO}_{2}$ film on Si. Figure 5(a) shows the Ru L $\alpha$ peak recorded during the very first cycles of the thermal ALD process on the $\mathrm{Si}-\mathrm{H}$ surface. The high sensitivity of the synchrotronbased XRF technique is illustrated by the fact that one single ALD cycle resulted in a clear fluorescence signal, implying submonolayer sensitivity. ${ }^{13,14}$ In Figure 5(b), the integrated $\mathrm{Ru} \mathrm{L} \alpha$ intensity is plotted against the number of deposition cycles for the thermal ALD process on $\mathrm{Si}-\mathrm{H}$ and $\mathrm{SiO}_{2}$, and for the plasma-enhanced ALD process on $\mathrm{SiO}_{2}$. Because the XRF intensity is proportional to the amount of $\mathrm{Ru}$ on the surface, the obtained growth curves provide direct information about the difference in deposition rate with process conditions and substrate nature. If one compares the results for 70 cycles of thermal ALD, Ru is deposited from the first cycle onward on $\mathrm{Si}-\mathrm{H}$, while no growth is observed on the $\mathrm{SiO}_{2}$ surface. These results are in line with our earlier findings based on in situ $\mathrm{SE}$ growth curves, and the easy nucleation on $\mathrm{Si}-\mathrm{H}$ can be explained by the oxidizing nature of the $\mathrm{RuO}_{4}$ molecule in combination with the oxidizable nature of the $\mathrm{Si}-\mathrm{H}$ surface. ${ }^{37}$ From Figure 5(b), it can furthermore be concluded that $\mathrm{H}_{2}$ (a)

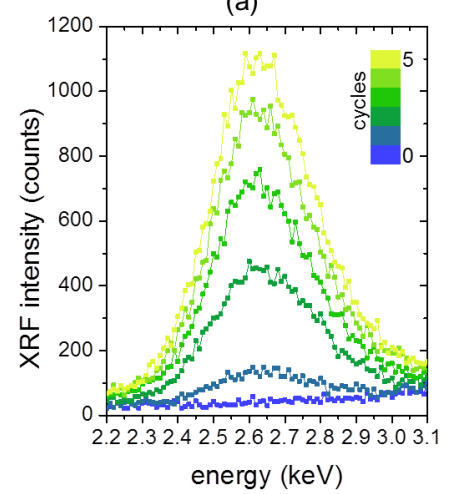

(b)

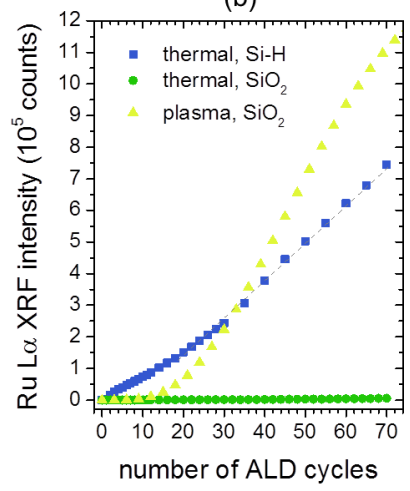

FIG. 5. (a) Evolution of the Ru L $\alpha$ fluorescence peak as measured during the first $5 \mathrm{ALD}$ cycles of the thermal $\mathrm{RuO}_{4} / \mathrm{H}_{2}$ process on a $\mathrm{Si}-\mathrm{H}$ surface. (b) Integrated $\mathrm{Ru} \mathrm{L} \alpha$ fluorescence intensity against the number of ALD cycles for the thermal $\mathrm{RuO}_{4} / \mathrm{H}_{2}$ process on $\mathrm{Si}-\mathrm{H}$ (blue squares) and on $\mathrm{SiO}_{2}$ (green circles), and for the plasma-enhanced $\mathrm{RuO}_{4} / \mathrm{H}_{2}$ plasma process on $\mathrm{SiO}_{2}$ (yellow triangles).

plasma helps to initiate nucleation on $\mathrm{SiO}_{2}$. The growth curve for the plasma-based ALD process shows a ca. 10 cycle growth delay and a pronounced S-shape that is indicative of island growth. $^{39}$

\section{B. In situ GISAXS}

GISAXS data were acquired using a 2D XPAD detector with a pixel size of $130 \times 130 \mu \mathrm{m}^{2} .{ }^{40}$ The sample-to-detector distance was set to ca. $50 \mathrm{~cm}$. Figure 6(a) shows a selection of GISAXS patterns measured during plasma-enhanced ALD

(a)

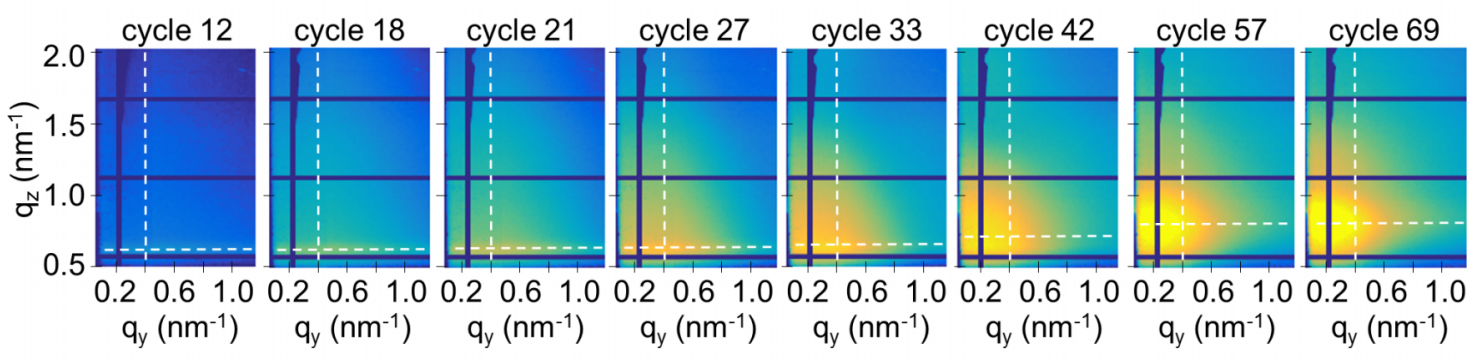

(b)

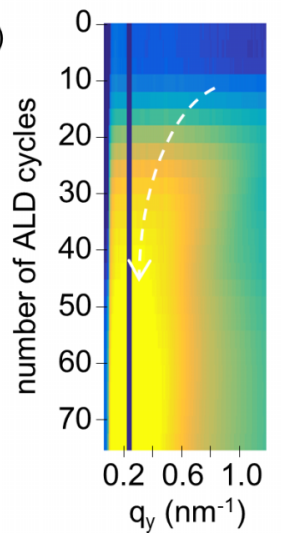

(c)

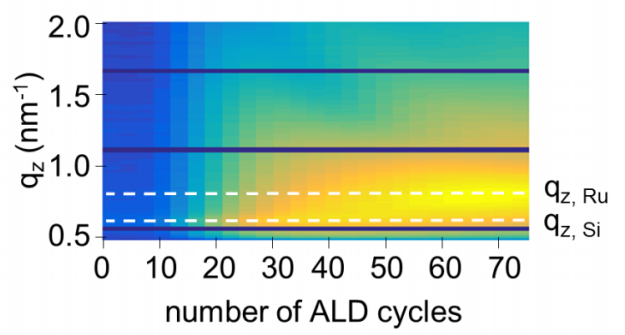

(d)

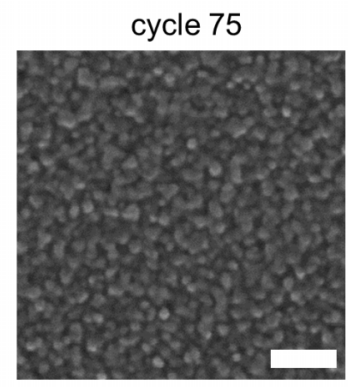

FIG. 6. (a) Selection of in situ recorded GISAXS patterns during the plasma-enhanced $\mathrm{RuO}_{4} / \mathrm{H}_{2}$ plasma process on $\mathrm{SiO}$. The dashed lines indicate the positions of the line profiles shown in (b) and (c). (b) 2D color map showing the intensity evolution of the horizontal line profile taken at the $\mathrm{q}_{z}$-position of maximum intensity. (c) $2 \mathrm{D}$ color map showing the intensity evolution of the vertical line profile taken at $\mathrm{q}_{\mathrm{y}}=0.4 \mathrm{~nm}^{-1}$. (d) SEM image with $100 \mathrm{~nm}$ scale bar of the Ru film after 75 ALD cycles. 
of $\mathrm{Ru}$ on $\mathrm{SiO}_{2}$. The complete image sequence recorded during the experiment is available as a movie (see the supplementary material). From ca. 10 ALD cycles, i.e., the onset of ALD growth as indicated by XRF, a clear side peak can be observed, which confirms the island-like growth mode. With progressing deposition, the scattering peak becomes more intense and shifts towards lower $\mathrm{q}_{\mathrm{y}}$-values and higher $\mathrm{q}_{\mathrm{z}}$-values. The evolution of the scattering features as a function of the number of ALD cycles is clearly visible in the $2 \mathrm{D}$ color plots in Figures 6(b) and 6(c), showing horizontal and vertical line profiles, respectively. The peak shift to lower $\mathrm{q}_{\mathrm{y}}$-values in Figure 6(b) indicates an increase in particle size and center-tocenter particle distance, which is probably due to static and/or dynamic coalescence of adjacent $\mathrm{Ru}$ islands. ${ }^{41}$ The position of maximum intensity also shifts to higher $\mathrm{q}_{\mathrm{z}}$-values when more $\mathrm{Ru}$ is deposited (Figure 6(c)). This is to be expected as a scattering enhancement is known to occur at an exit angle which equals the critical angle of the sample (Yoneda peak). ${ }^{30}$ Because the critical angle at $5 \mathrm{keV}$ is higher for $\mathrm{Ru}$ $\left(\alpha_{\text {crit, Ru }}=0.764^{\circ}\right)$ than for $\mathrm{Si}\left(\alpha_{\text {crit }, \mathrm{Si}}=0.361^{\circ}\right)$, the scattering enhancement appears at higher exit angles, and thus higher $\mathrm{q}_{\mathrm{z}}$-values, when the $\mathrm{SiO}_{2}$ surface gets more and more covered with Ru. A scanning electron microscopy (SEM) image taken after the deposition confirms a particulated Ru morphology (Figure 6(d)).

Figure 7(b) shows the GISAXS pattern measured after 70 thermal ALD cycles on $\mathrm{Si}-\mathrm{H}$. Based on the XRF intensity, the selected GISAXS pattern agrees in Ru loading with the pattern plotted in Figure 7(a) for the plasma-enhanced ALD process on $\mathrm{SiO}_{2}$. Both patterns also use the same color intensity (a)
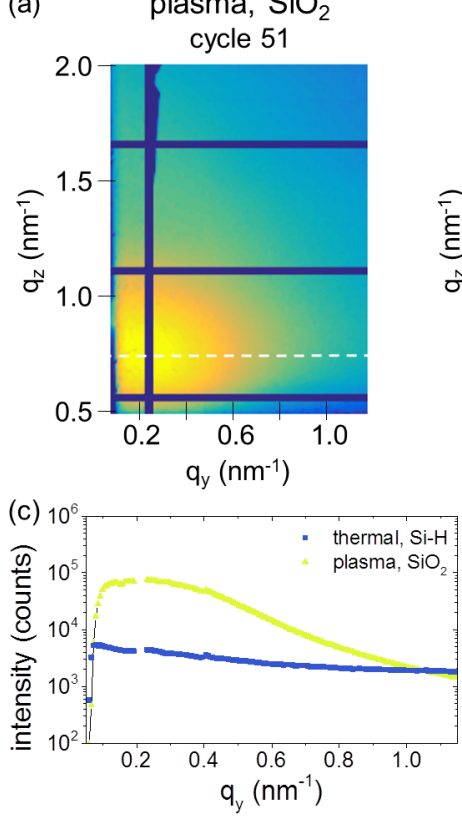

(b) thermal, Si-H cycle 70

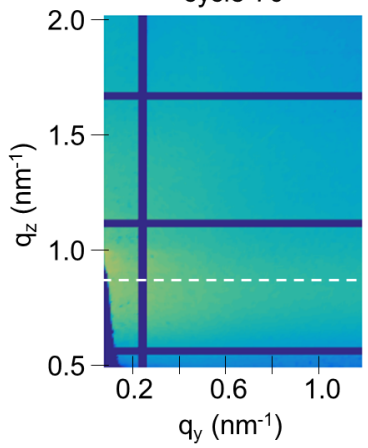

(d)

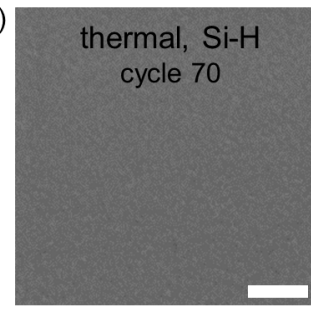

FIG. 7. (a) and (b) In situ recorded GISAXS patterns measured after (a) 51 ALD cycles of the plasma-enhanced $\mathrm{RuO}_{4} / \mathrm{H}_{2}$ plasma process on $\mathrm{SiO}_{2}$ and (b) $70 \mathrm{ALD}$ cycles of the thermal $\mathrm{RuO}_{4} / \mathrm{H}_{2}$ process on a $\mathrm{Si}-\mathrm{H}$ surface. The dashed lines indicate the positions of the line profiles shown in (c). (c) Comparison of the horizontal line profiles taken at the $\mathrm{q}_{\mathrm{z}}$-position of maximum intensity. (d) SEM image with $100 \mathrm{~nm}$ scale bar of the Ru film grown after 70 ALD cycles of the thermal $\mathrm{RuO}_{4} / \mathrm{H}_{2}$ process on a $\mathrm{Si}-\mathrm{H}$ surface. scale, and Figure 7(c) compares the horizontal line profiles. It can be concluded that the overall scattered intensity is much lower for thermal ALD growth on $\mathrm{Si}-\mathrm{H}$ (Figure 7(b)) than for plasma-enhanced ALD growth on $\mathrm{SiO}_{2}$ (Figure 7(a)). Also, no clear scattering maxima are present in the $2 \mathrm{D}$ pattern shown in Figure 7(b), nor in any of the preceding 2D GISAXS patterns that were measured in situ during the thermal process on $\mathrm{Si}-\mathrm{H}$ (see movie in the supplementary material). This suggests that on $\mathrm{Si}-\mathrm{H} \mathrm{Ru}$ starts to grow as a continuous layer from the start. The visible scattering results from an increase in overall surface roughness with Ru deposition. ${ }^{14}$ After 70 ALD cycles, the Ru layer appeared smooth in SEM (Figure 7(d)).

\section{v. CONCLUSIONS}

A pump-type plasma-enhanced ALD reactor was designed to be compatible with different synchrotron beamlines (SixS and SAMBA at SOLEIL and DUBBLE at the ESRF) to enable in situ characterization of the deposition process with XRF, GISAXS, and XAS. During experiments in the lab and transport to the synchrotron, the system is installed on a mobile support rack. At the synchrotron, the compact design enables quick and easy installation by lifting the complete setup onto the diffractometer. The use of interchangeable vertical side flanges offers a large flexibility when designing experiments: dedicated sample heaters (horizontal or vertical), $\mathrm{x}$-ray transparent windows and anti-scattering systems, or quartz windows can be mounted to obtain the desired measurement geometry.

The setup was used to monitor thermal and plasmaenhanced ALD growth of ruthenium using $\mathrm{RuO}_{4}$ as precursor and $\mathrm{H}_{2}$ or $\mathrm{H}_{2}$ plasma as reactant. Based on the obtained XRF growth curves and qualitative analysis of the 2D GISAXS patterns, it is concluded that $\mathrm{Ru}$ grows as a smooth layer and without incubation on $\mathrm{H}$-terminated $\mathrm{Si}$. On $\mathrm{SiO}_{2}$, no growth was observed during a 70 cycle deposition with the thermal process, while for plasma-enhanced ALD of $\mathrm{Ru}$, a nucleationcontrolled growth is observed marked by the formation of $\mathrm{Ru}$ islands following a 10 cycle incubation time.

\section{SUPPLEMENTARY MATERIAL}

See the supplementary material for the full GISAXS image sequences of the experiments.

\section{ACKNOWLEDGMENTS}

J.D., M.M.M., and K.V.d.K. acknowledge the FWOVlaanderen for financial support through a personal research grant. The authors also acknowledge BOF-UGent (Grant No. GOA 01G01513), the Hercules foundation, and FWOVlaanderen for providing funding for this work. The authors thank Professor J. Haemers and Dr. G. Rampelberg for their valuable contributions to the design of the chamber, $\mathrm{L}$. Tassignon for the CAD drawings, S. Broekaert and J. Sys for their help with the mechanical and electrical construction of the chamber, and Dr. K. De Keyser for his help concerning the control software. We are also grateful to the SOLEIL 
and ESRF staff for smoothly running the synchrotron and beamline facilities and to Christian Dussarrat (Air Liquide) for providing the ToRuS precursor.

${ }^{1}$ R. L. Puurunen, J. Appl. Phys. 97, 121301 (2005).

${ }^{2}$ S. M. George, Chem. Rev. 110, 111 (2010).

${ }^{3}$ C. Detavernier, J. Dendooven, S. P. Sree, K. F. Ludwig, and J. A. Martens, Chem. Soc. Rev. 40, 5242 (2011).

${ }^{4}$ K. J. Kanarik, T. Lill, E. A. Hudson, S. Sriraman, S. Tan, J. Marks, V. Vahedi, and R. A. Gottscho, J. Vac. Sci. Technol., A 33, 020802 (2015).

${ }^{5}$ H. B. Profijt, S. E. Potts, M. C. M. van de Sanden, and W. M. M. Kessels, J. Vac. Sci. Technol., A 29, 050801 (2011).

${ }^{6}$ K. Knapas and M. Ritala, Crit. Rev. Solid State Mater. Sci. 38, 167 (2013).

${ }^{7}$ J. Knudsen, J. N. Andersen, and J. Schnadt, Surf. Sci. 646, 160 (2016).

${ }^{8}$ A. R. Head, S. Chaudhary, G. Olivieri, F. Bournel, J. N. Andersen, F. Rochet, J.-J. Gallet, and J. Schnadt, J. Phys. Chem. C 120, 243 (2016).

${ }^{9}$ E. Langereis, S. B. S. Heil, H. C. M. Knoops, W. Keuning, M. C. M. van de Sanden, and W. M. M. Kessels, J. Phys. D: Appl. Phys. 42, 073001 (2009).

${ }^{10}$ J. Dendooven, D. Deduytsche, S. P. Sree, T. Korányi, G. Vanbutsele, J. A. Martens, K. F. Ludwig, and C. Detavernier, in AVS Topical Conference on ALD, Seoul, South Korea, 2010.

${ }^{11}$ D. D. Fong, J. A. Eastman, S. K. Kim, T. T. Fister, M. J. Highland, P. M. Baldo, and P. H. Fuoss, Appl. Phys. Lett. 97, 191904 (2010).

${ }^{12}$ W. Setthapun, W. D. Williams, S. M. Kim, H. Feng, J. W. Elam, F. A. Rabuffetti, K. P. Poeppelmeier, P. C. Stair, E. A. Stach, F. H. Ribeiro, J. T. Miller, and C. L. Marshall, J. Phys. Chem. C 114, 9758 (2010).

${ }^{13}$ J. Dendooven, S. P. Sree, K. De Keyser, D. Deduytsche, J. A. Martens, K. F. Ludwig, and C. Detavernier, J. Phys. Chem. C 115, 6605 (2011).

${ }^{14}$ K. Devloo-Casier, J. Dendooven, K. F. Ludwig, G. Lekens, J. D’Haen, and C. Detavernier, Appl. Phys. Lett. 98, 231905 (2011).

${ }^{15}$ Y. J. Park, D. R. Lee, H. H. Lee, H.-B.-R. Lee, H. Kim, G.-C. Park, S.-W. Rhee, and S. Baik, J. Nanosci. Nanotechnol. 11, 1577 (2011).

${ }^{16}$ Y. Lei, J. Lu, H. Zhao, B. Liu, K.-B. Low, T. Wu, J. A. Libera, J. P. Greeley, P. J. Chupas, J. T. Miller, and J. W. Elam, J. Phys. Chem. C 117, 11141 (2013).

${ }^{17}$ K. Devloo-Casier, K. F. Ludwig, C. Detavernier, and J. Dendooven, J. Vac. Sci. Technol., A 32, 010801 (2014).

${ }^{18}$ J. Dendooven, K. Devloo-Casier, M. Ide, K. Grandfield, M. Kurttepeli, K. F. Ludwig, S. Bals, P. Van der Voort, and C. Detavernier, Nanoscale 6, 14991 (2014).

${ }^{19}$ M. Filez, H. Poelman, R. K. Ramachandran, J. Dendooven, K. DevlooCasier, E. Fonda, C. Detavernier, and G. B. Marin, Catal. Today 229, 2 (2014).

${ }^{20}$ S. M. Geyer, R. Methaapanon, R. W. Johnson, W.-H. Kim, D. G. Van Campen, A. Metha, and S. F. Bent, Rev. Sci. Instrum. 85, 055116 (2014).

${ }^{21}$ J. A. Klug, M. S. Weimer, J. D. Emery, A. Yanguas-Gil, S. Seifert, C. M. Schlepütz, A. B. F. Martinson, J. W. Elam, A. S. Hock, and T. Proslier, Rev. Sci. Instrum. 86, 113901 (2015).
${ }^{22}$ R. Boichot, L. Tian, M.-I. Richard, A. Crisci, A. Chaker, V. Cantelli, S. Coindeau, S. Lay, T. Ouled, C. Guichet, M. H. Chu, N. Aubert, G. Ciatto, E. Blanquet, O. Thomas, J.-L. Deschanvres, D. D. Fong, and H. Renevier, Chem. Mater. 28, 592 (2016).

${ }^{23}$ K. Devloo-Casier, P. Geiregat, K. F. Ludwig, K. van Stiphout, A. Vantomme, Z. Hens, C. Detavernier, and J. Dendooven, J. Phys. Chem. C 120, 18039 (2016).

${ }^{24}$ G. Ozaydin, A. S. Ozcan, Y. Wang, K. F. Ludwig, H. Zhou, R. L. Headrick, and D. P. Siddons, Appl. Phys. Lett. 87, 163104 (2005).

${ }^{25}$ M. Tallarida, K. Karavaev, and D. Schmeisser, J. Appl. Phys. 104, 064116 (2008).

${ }^{26}$ M. Tallarida and D. Schmeisser, Semicond. Sci. Technol. 27, 074010 (2012).

${ }^{27}$ R. Methaapanon, S. M. Geyer, C. Hagglund, P. A. Pianetta, and S. F. Bent, Rev. Sci. Instrum. 84, 015104 (2013).

${ }^{28}$ S. M. Geyer, R. Methaapanon, B. Shong, P. A. Pianetta, and S. F. Bent, J. Phys. Chem. Lett. 4, 176 (2013).

${ }^{29}$ E. Levrau, K. Devloo-Casier, J. Dendooven, K. F. Ludwig, P. Verdonck, J. Meersschaut, M. R. Baklanov, and C. Detavernier, Langmuir 29, 12284 (2013).

${ }^{30}$ G. Renaud, R. Lazzari, and F. Leroy, Surf. Sci. Rep. 64, 255 (2009).

${ }^{31}$ S. T. Christensen, J. W. Elam, B. Lee, Z. Feng, M. J. Bedzyk, and M. C. Hersam, Chem. Mater. 21, 516 (2009).

${ }^{32}$ J. Dendooven, K. Devloo-Casier, A. Coati, G. Portale, W. Bras, K. F. Ludwig, and C. Detavernier, in 12th International Conference on SolidState and Integrated Circuit Technology, ICSICT '14, Guilin, China, 28-31 October 2014 (IEEE Conference Publications, 2014).

${ }^{33}$ G. Portale, D. Cavallo, G. C. Alfonso, D. Hermida-Merino, M. van Drongelen, L. Balzano, G. W. M. Peters, J. G. P. Goossens, and W. Bras, J. Appl. Crystallogr. 46, 1681 (2013).

${ }^{34}$ V. Briois, E. Fonda, S. Belin, L. Barthe, C. La Fontaine, F. Langlois, M. Ribbens, and F. Villain, UVX 2010; Applications et Développements Récents (EDP Sciences, 2011), pp. 41-47.

${ }^{35}$ S. M. Rossnagel, A. Sherman, and F. Turner, J. Vac. Sci. Technol., B 18, 2016 (2000).

${ }^{36}$ J. Dendooven, R. K. Ramachandran, K. Devloo-Casier, G. Rampelberg, M. Filez, H. Poelman, G. B. Marin, E. Fonda, and C. Detavernier, J. Phys. Chem. C 117, 20557 (2013).

${ }^{37}$ M. M. Minjauw, J. Dendooven, B. Capon, M. Schaekers, and C. Detavernier, J. Mater. Chem. C 3, 132 (2015).

${ }^{38}$ M. M. Minjauw, J. Dendooven, B. Capon, M. Schaekers, and C. Detavernier, J. Mater. Chem. C 3, 4848 (2015).

${ }^{39}$ R. L. Puurunen and W. Vandervorst, J. Appl. Phys. 96, 7686 (2004).

${ }^{40}$ C. Mocuta, M.-I. Richard, J. Fouet, S. Stanescu, A. Barbier, C. Guichet, O. Thomas, S. Hustache, A. V. Zozulya, and D. Thiaudière, J. Appl. Crystallogr. 46, 1842 (2013).

${ }^{41}$ M. Schwartzkopf, A. Buffet, V. Körstgens, E. Metwalli, K. Schlage, G. Benecke, J. Perlich, M. Rawolle, A. Rothkirch, B. Heidmann, G. Herzog, P. Müller-Buschbaum, R. Röhlsberger, R. Gehrke, N. Stribeck, and S. V. Roth, Nanoscale 5, 5053 (2013). 